\title{
Cosmic Sparks from Superconducting Strings
}

\author{
Tanmay Vachaspati \\ Institute for Advanced Study, Princeton, NJ 08540. \\ CERCA, Physics Department, Case Western Reserve University, \\ 10900 Euclid Avenue, Cleveland, OH 44106-7079.
}

\begin{abstract}
We investigate cosmic sparks from cusps on superconducting cosmic strings in light of the recently discovered millisecond radio burst by Lorimer et al [1]. We find that the observed duration, fluence, spectrum, and event rate can be reasonably explained by Grand Unification scale superconducting cosmic strings that carry currents $\sim 10^{5} \mathrm{GeV}$. The superconducting string model predicts an event rate that falls off only as $S^{-1 / 2}$, where $S$ is the energy flux, and hence predicts a population of very bright bursts. Other surveys, with different observational parameters, are shown to impose tight constraints on the superconducting string model.
\end{abstract}

The discovery of a radio burst ("spark") was recently reported by Lorimer et al [1] in the Parkes survey and analysis of the dispersion measure suggests that the source is of cosmological origin. A thorough examination of the observation has supported this conclusion [2]. Conventional astrophysical sources are not known and neither has a host galaxy for the event been identified.

If more cosmic sparks are observed and found to be extra-galactic, it would indicate an exotic cosmological process. Superconducting cosmic strings are a possible exotic source of electromagnetic phenomena in the universe and arise quite naturally in particle physics models [3], though less so in string theory [4]. Earlier work on cosmological signatures of superconducting cosmic strings has primarily foucussed on high energy emission in the form of particles and/or gamma rays [3, 5, 6] or synchrotron emission [7]. In this paper we argue that radio emission may be a good way to look for superconducting strings as they can cause observable sparks similar to the one seen by Lorimer et al. Even if further observations discover astrophysical sources for observed sparks, the prediction that superconducting cosmic strings produce radio sparks that are potentially observable is important from the particle physics viewpoint, since their detection or absence may be used to constrain various fundamental models.

Superconducting cosmic strings can be viewed as elastic, current-carrying wires, distributed in the cosmos as closed loops and infinitely long Brownian curves. The mass per unit length of a string will be denoted by $\mu=\eta^{2}$ and the current by $i_{0}$. Current-carrying strings oscillate under their own tension and radiate electromagnetically (e.g. see [8]). The radiation is very strong from events such as "cusps" which are points on an idealized (zero thickness, no current) string that reach the speed of light for a brief instant [9, 10, 11]. In a more realistic setting, the cusp gets cut-off due to the thickness of the string and due to the backreaction of the current and radiation. Nonetheless the radiation is very strong from localized regions ("quasi-cusps") even in the realistic string case. The scenario we envision is that a curved section of string (or loop) of length $L$ develops a cusp and beams

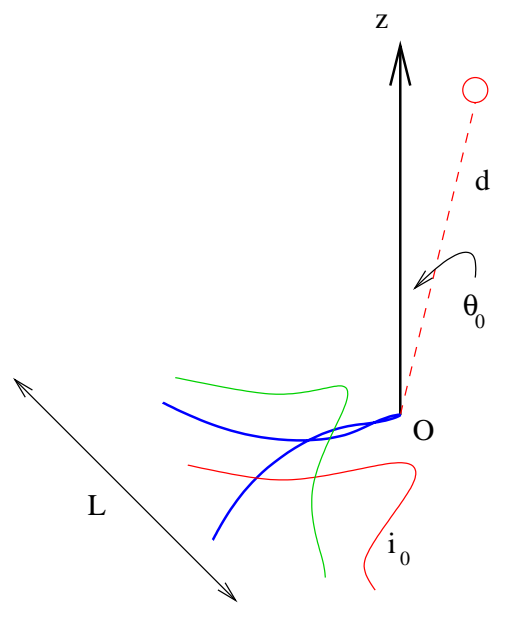

FIG. 1: String segment with current $i_{0}$ at 3 different times, with a cusp at $\mathrm{O}$, with velocity along $z$. The size of the curved section of string is $L$. The observer is at an angle $\theta_{0}$ from the z-axis at a distance $d$.

electromagnetic radiation in direction $\hat{z}$. The observer is located at some large distance, $d$, from the location of the cusp and slightly off the $z$ axis, at an angle $\theta_{0}$ (Fig. 1).

The energy emitted from a cusp at angular frequency $\omega=2 \pi \nu$, at angle $\theta$ from the beam direction, and observed at distance $d$ (i.e. the fluence) is given by

$$
F \equiv \frac{1}{d^{2}} \frac{d^{2} E_{\omega}}{d \omega d \Omega} \sim b i_{0}^{2} \frac{L^{2}}{d^{2}} e^{-a \omega L \theta^{3}} \quad \text { if } \quad a \omega L \theta^{3}>1
$$

where $a$ and $b$ are constants that depends on the shape of the cusp and we will take $a \sim 1, b \sim 1$. (We also work in natural units throughout, so $\hbar=1=c$.) This result is most easily obtained by expanding the result in Ref. 10] for large $\omega L \theta^{3}$. If, instead, we have $\omega L \theta^{3}<1$, the emitted energy falls off $\propto \omega^{-2 / 3}$.

The total energy emitted from a loop in electromagnetic and gravitational radiation in one time period is $E_{\text {total,loop }} \sim\left(\kappa e i_{0} \eta+\Gamma G \mu^{2}\right) L \equiv \gamma \mu L$ where $e \approx 0.3$ is the charge of the current carriers, $G$ Newton's gravitational constant, and $\kappa \sim 30, \Gamma \sim 100$ are numerical factor determined by averaging numerically derived radi- 
ation rates from a variety of loops.

The temporal width of the emission seen from the cusp is given by the duration for which the observer is in the beam. The emission from the cusp is beamed but the beam is changing direction at a rate $\dot{\theta} \sim 1 / L$. So the beam changes its direction by $\theta_{0}$ in a time $L \theta_{0}$. Since, for large angles from the beam, the emission gets cutoff exponentially fast (Eq. (1D)), we have $\theta_{0} \sim(\omega L)^{-1 / 3}$, and

$$
\Delta t \sim \omega^{-1 / 3} L^{2 / 3}
$$

The event rate depends on the number density of loops and is derived from numerical simulations of nonsuperconducting strings [8]. Note that the results for non-superconducting strings should also apply in the case that the current on the string is much smaller than the symmetry breaking scale $\eta$. The simulations ignore radiative effects and find that the number density of loops of length between $l_{0}$ and $l_{0}+d l_{0}$ is $d n_{l_{0}} \sim A d l_{0} /\left(l_{0}^{2} t^{2}\right)$ where $A \sim 10^{2}$ [12, 13, 14] (also see 15]). Due to radiation, the length $l$ of a loop decreases with time, $l(t)=l_{0}-\gamma\left(t-t_{i}\right)$, where $t_{i}$ is the time when the loop was born. So the number density of loops of size $l$ at time $t$ is

$$
d n_{l}(t) \sim \frac{A d l}{(l+\gamma t)^{2} t^{2}}
$$

where the radiative shrinking has been included, and we have assumed that the loops were all born at some very early time so that $t \gg t_{i}$.

The spark observed by Lorimer et al was in the frequency interval $(1.28,1.52) \mathrm{GHz}$ and the central frequency is $\nu_{0}=1.4 \mathrm{GHz}$. Based on the dispersion measure of the event, the observed event is constrained to lie within redshift of $0.1-0.3$ and, for our estimates, we will assume that the event was located at $z_{0}=0.3$, or at a comoving distance $\sim 1 \mathrm{Gpc}$. The observed energy flux is

$$
S_{\text {obs }}=30 \mathrm{Jy}=3 \times 10^{-22} \frac{\text { ergs }}{\mathrm{cm}^{2}-\mathrm{s}-\mathrm{Hz}}
$$

and the duration of the event has an upper bound $\Delta \tau \lesssim$ $\Delta \tau_{0}=5 \mathrm{~ms}$

The observed pulse width cannot be used to estimate the intrinsic duration of the event because of scattering by the turbulent inter-galactic medium (IGM) [1, 2]. The oberved time width, $\Delta t_{\text {obs }}$, is a sum in quadratures of the intrinsic time width modified by cosmological redshift and the width due to scattering in the IGM: $\Delta t_{\mathrm{obs}}=$ $\left[\left(\Delta \tau_{\mathrm{obs}}\right)^{2}+\left(\Delta t_{\mathrm{emit}}\right)^{2}\right]^{1 / 2}$ with

$$
\Delta t_{\mathrm{emit}}=(1+z) \omega_{\mathrm{emit}}^{-1 / 3} l^{2 / 3}=\omega_{0}^{-1 / 3}(1+z)^{2 / 3} l^{2 / 3}
$$

The scattering time width at frequency $\nu$ from an event at redshift $z$ depends on the location of the scattering centers. For the case of a scattering screen close to the source, the width is [2, 19]

$$
\Delta \tau_{\mathrm{obs}}(\nu, z)=\Delta \tau_{0}\left(\frac{1+z}{1+z_{0}}\right)^{\beta+1}\left(\frac{\nu}{\nu_{0}}\right)^{\beta}
$$

where $\Delta \tau_{0}=5 \mathrm{~ms}, \nu_{0}=1.4 \mathrm{GHz}, z_{0}=0.3$, and $\beta=-4.8$. For comparison with the observed spark in the Parkes survey, we will use $\nu=\nu_{0}$, but if comparing to other surveys it will be necessary to insert the appropriate observational frequency.

The observed power law fall off $\propto \nu^{-4}$ in the observational frequency band can be fit by an exponential, and since the fluence is $S_{\mathrm{obs}} \Delta t_{\mathrm{obs}}$,

$$
\begin{aligned}
F_{\text {obs }} & =\frac{S_{\text {obs }}}{2 \pi} \Delta \tau_{0} \sqrt{1+\left(\frac{\Delta t_{0}}{\Delta \tau_{0}}\right)^{2}} e^{-\left(\nu-\nu_{0}\right) / \nu_{c}} \\
& \approx 10^{-23} e^{-4 \nu / \nu_{0}} \frac{\mathrm{ergs}}{\mathrm{cm}^{2}-\mathrm{Hz}}
\end{aligned}
$$

To get an idea of the parameters needed of the superconducting string model, we first fit the observed spectrum ignoring redshift factors (which are small since $z_{0}<0.3$ ). Using Eq. (1) we find

$$
\frac{d \ln F}{d \ln \omega}=-a \omega L \theta^{3}=-4
$$

The observation does not constrain the intrinsic duration of the event. However, if the intrinsic duration was $1 \mathrm{~ms}$, we can equate the duration of the cusp event (Eqs. (2)) to the observed duration to get $\omega_{0}^{-1 / 3} L^{2 / 3}=$ $\Delta t_{0}=1 \mathrm{~ms}$, where $\omega_{0}=2 \pi \nu_{0} \sim 10^{10} \mathrm{~s}^{-1}$. This gives $L=\omega_{0}^{1 / 2}(\Delta t)^{3 / 2} \sim 3 \mathrm{~s}=10^{11} \mathrm{~cm}$. If the intrinsic duration were smaller than $1 \mathrm{~ms}$, the length would be even smaller. This illustrates that we are considering signatures of loops that are very small on a cosmological scale.

The energy flux from the cusp event is found from Eqs. (1) and (8). Equating to the observed value, Eq. (7), gives

$$
i_{0} \sim 10^{5}\left(\frac{10^{11} \mathrm{~cm}}{L}\right)\left(\frac{d}{1 \mathrm{Gpc}}\right) \mathrm{GeV}
$$

where we have used $1 \mathrm{ergs} /\left(\mathrm{cm}^{2}-\mathrm{Hz}\right)=1 \mathrm{GeV}^{2}$. Note also that the dynamics of the string will be dominated by the tension if $\eta \gg 10^{6} \mathrm{GeV}$. Hence it is a valid approximation to ignore the current when discussing the dynamics of the string network.

To estimate the rate of observed-sparker-like events, we use Eq. (3). Then the event rate, denoted $\dot{N}$, due to cusps on loops of size between $l$ and $l+d l$, beamed within a solid angle $d \Omega$ from us is

$$
d \dot{N} \sim \frac{f_{c}}{l} \frac{A d l}{\left(l+\gamma t_{0}\right)^{2} t_{0}^{2}} \frac{d \Omega}{4 \pi} d V
$$

where the factors account for $f_{c}$ cusps per loop oscillation, the number density of loops, the beaming angle constraint, and the spatial volume. For Grand Unification scale strings, $\eta \sim 10^{14} \mathrm{GeV}$, we find $\gamma t_{0} \sim 10^{9} \mathrm{~s} \gg$ $l \sim 1 \mathrm{~s}$, and we can rewrite (10) as

$$
d \dot{N} \sim C \times \frac{d l}{l} \times \sin \theta d \theta \times D^{2} d D
$$


where $D \equiv H_{0} D_{c}$ is the comoving distance to the loop in Hubble units, and $C \equiv 2 \pi A f_{c} H_{0} / \gamma^{2} \sim 10^{7} f_{c}$ day $^{-1}$, for $\gamma=10^{-8}$. Now that we know the rate of events as a function of $l, \theta$ and $D_{c}$, we can also evaluate the flux density in these terms

$$
S\left(l, \theta, D_{c}\right)=\frac{2 \pi i_{0}^{2} l^{2}}{D_{c}^{2}(\Delta t)_{\mathrm{obs}}} e^{-\omega_{0}(1+z) l \theta^{3}}, \omega_{0}(1+z) l \theta^{3}>1
$$

and we assume a "top hat" cut-off at small angles, $S\left(l, \theta, D_{c}\right)=2 \pi e^{-1} i_{0}^{2} l^{2} / D_{c}^{2}(\Delta t)_{\text {obs }}$ if $\omega_{0}(1+z) l \theta^{3} \leq 1$.

The event rate relevant to a survey is

$$
\dot{N}(>S)=C \int_{\mathcal{V}} \frac{d l}{l} D^{2} d D \sin \theta d \theta
$$

Here the integration volume $\mathcal{V}$ is constrained by the requirement that the flux be greater than $S$ and that the observed duration of the event, $(\Delta t)_{\mathrm{obs}}$, be in the range in which the search was carried out. In our case, $(\Delta t)_{\text {obs }}$ is in the interval $(1 \mathrm{~ms}, 1 \mathrm{~s})$ to coincide with the search parameters in Ref. [1], and we will assume a standard flat cosmology. After integrating over $\theta$ by hand, the remaining integrals were evaluated numerically, giving

$$
\dot{N}(>30 \mathrm{Jy}) \sim 10^{0} f_{c} \text { day }^{-1}
$$

whereas the single observed event gives a $99 \%$ doublesided confidence level estimate between 2 to $3 \times 10^{3}$ events per day [2]. (Based on the dynamics of smooth loops, one expects $f_{c} \sim 1$.) The absence of fainter events, but still above the threshold of $0.3 \mathrm{Jy}$, is not significant since the prediction is only around 10 events above $0.3 \mathrm{Jy}$, and also the reach of the Parkes survey at threshold is less by a factor $\sim 10$ than at $30 \mathrm{Jy}[2$. This shows that the superconducting string model can give an event rate consistent with the Lorimer et al observations. Further, it is possible to show that for fixed observational frequency and duration range, $\dot{N}(>S) \propto S^{-1 / 2}$ for large $S$. This is quite different from the $S^{-3 / 2}$ fall off expected from uniformly distributed, identical sources. The slow fall off implies that there may be a population of very strong sparks e.g. $\sim 1$ per year above $1 \mathrm{MJy}$.

A more thorough analysis, taking into account the constraints imposed by different surveys, may impose further limits on the model. For example, the STARE survey 20] places an upper bound of $7.5 \times 10^{-2}$ events per day [2] for $S>80 \mathrm{kJy}$ at an observational frequency of $611 \mathrm{MHz}$ and durations ranging from $125 \mathrm{~ms}$ to a few minutes. With these observational parameters and the superconducting string model parameters used above, the model prediction is $\sim 6 \times 10^{-2}$ events per day. This suggests that useful constraints on the string model may be placed by presently existing data (see Fig. 22). In particular, a value of $\gamma$ significantly less than $10^{-8}$ is already ruled out.

While we expect a quasi-cusp to repeat every oscillation, we do not expect the beaming direction to remain

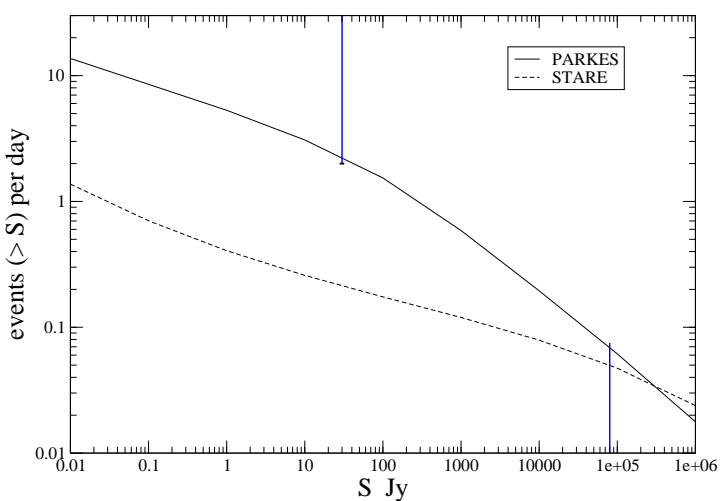

FIG. 2: Event rates for PARKES and STARE survey parameters. The vertical line at $30 \mathrm{Jy}$ corresponds to the inferred rate from the PARKES survey and at 80000 Jy shows the constraint from the STARE survey.

the same in every repetition. The angular momentum carried off by the beamed radiation is $\sim E_{\text {beam }} L$ and the moment of inertia of the string in the cusp region is $\sim(\mu \sigma) L^{2}$, where $\sigma \sim\left(\omega_{0} L\right)^{-1 / 3} L$ is the length of string in the cusp region relevant to the observed radiation frequency. The angular velocity due to radiation backreaction is $\dot{\theta} \sim E_{\text {beam }} L / \mu \sigma L^{2}$. Since the time interval between two cusp events is $\sim L$, we estimate the change in the beaming angle as

$$
\Delta \theta \sim \frac{E_{\text {beam }} L}{\mu \sigma L^{2}} L \sim \frac{\kappa e i_{0}}{\eta}\left(\omega_{0} L\right)^{1 / 3} \sim 2 \times 10^{-4}
$$

which corresponds to a change in the observed flux $|\Delta \ln F| \sim\left|a \omega_{0} L \theta_{0}^{3}\right| \times 3 \Delta \theta / \theta_{0} \sim 5$ Hence the beamed fluence may get reduced by a factor $e^{-5} \sim 10^{-2}$. This estimate suggests that sparks due to consecutive cusps on strings would be beamed in only slightly different directions but, since the fluence is highly sensitive to the beaming direction (Eq. (1)), the repeated event may or may not be observed, depending on the precise cusp shape and other parameters. In the 20 days that Lorimer et al searched for bursts, the particular loop would have on order $10^{6}$ cusps, and since the solid angle of the beam is order $10^{-6}$, we can expect on order one event in those 20 days. Also, cusps that occur much later, will occur with different parameters since the length and shape of the loop are likely to change due to backreaction.

To model the observations, we have taken strings whose tension scale is $\eta \sim 10^{14} \mathrm{GeV}$ (so that $\gamma \sim 10^{-8}$ ) but with currents of only $\sim 10^{5} \mathrm{GeV}$. The small current compared to the tension scale can have a natural origin in terms of scattering of counter-propagating particles on the string 21, 22] and/or the modified dispersion relation due to background magnetic fields [23].

We have considered only the direct emission of 1.4 
$\mathrm{GHz}$ radiation from the cusp. This corresponds to a very high harmonic emitted from the oscillating loop. The fundamental frequency is given by $L^{-1} \sim 1 \mathrm{~Hz}$, and $1 \mathrm{GHz}$ emission corresponds to the $10^{9}$ th harmonic. What happens to emission at lower frequencies? Since the cusp event is not expected to be in a host galaxy, it is surrounded only by the IGM with free electron number density $n_{e} \sim 10^{-7} \mathrm{~cm}^{-3}$ and plasma frequency $\omega_{p}=\left(4 \pi n_{e} e^{2} / m_{e}\right)^{1 / 2} \sim 30 \mathrm{~s}^{-1}$ or $\nu_{p} \sim 5 \mathrm{~Hz}$. The energy emitted from the cusp at $\nu \lesssim 5 \mathrm{~Hz}$ cannot propagate in the IGM and must push it around, creating shocks, fireballs, and possibly gamma ray bursts [5, 6, 24]. Assuming that all the very low frequency emission gets converted into gamma rays due to plasma effects, the power emitted is $\sim i_{0}^{2}=10^{10} \mathrm{GeV}^{2}$. For gamma rays at $1 \mathrm{GeV}$ and for loops at a distance of $1 \mathrm{Gpc}$, this gives a photon flux of $10^{-22} \mathrm{~cm}^{-2} \mathrm{~s}^{-1}$ which is far below the threshold, $\sim 10^{-8} \mathrm{~cm}^{-2} \mathrm{~s}^{-1}$ of the Third Interplanetary Network.

The electromagnetic emission from strings also distorts the cosmic microwave background spectrum and this effect has been used to constrain superconducting strings [24, 25]. Our choice of parameters, $G \mu \ll 10^{-6}$, is within these constraints. It is worth pointing out that the earlier work on gamma ray bursts from cusp events primarily focussed on superconducting strings placed in the galactic environment. More detailed investigation is needed to determine if gamma ray bursts are expected to accompany radio sparks occurring in the IGM.

The superconducting string model may be tested in a variety of ways. Gravitational effects of strings with $G \mu \sim 10^{-10}$ will be weak and may not be within forseeable detection capabilities, except possibly for gravitational wave bursts from cusps [26]. A promising possibility is to look for signatures of particle emission, such as positrons [27] or other decaying particles produced where the current on the string quenches. These particles would give a distinctive feature in the emission from the vicinity of the spark. In addition to the observed sparker like events, there should be rarer events where we are even closer to the beam, such that $\omega L \theta^{3}<1$. Then the spectrum will not decay exponentially, and the characteristics of the event should be quite different. Also, kinks on superconducting strings will radiate in unusual "fan-like" patterns [28]. Most immediately, it is necessary to find more sparks and check if they are associated with host galaxies since supermassive strings of the kind we have considered are expected to roam outside of galaxies.

I am grateful to Mark Hindmarsh, Mario Juric, Shri Kulkarni, Ken Olum, Jerry Ostriker, Edward Witten and Zheng Zheng for discussions. This work was supported in part by the U.S. Department of Energy and NASA at Case Western Reserve University.

[1] D. R. Lorimer, M. Bailes, M. A. McLaughlin, D. J. Narkevic and F. Crawford, arXiv:0709.4301 [astro- $\mathrm{ph}]$.

[2] S.R. Kulkarni, E.O. Ofek, J.D. Neill, M. Juric and Z. Zheng, "Giant Sparks at Cosmological Distances," unpublished (2007).

[3] E. Witten, Nucl. Phys. B 249, 557 (1985).

[4] J. Polchinski, arXiv:hep-th/0412244

[5] A. Babul, B. Paczynski and D. Spergel, Ap. J. Lett. 316, L49 (1987)

[6] V. Berezinsky, B. Hnatyk and A. Vilenkin, Phys. Rev. D 64, 043004 (2001)

[7] E. M. Chudnovsky, G. B. Field, D. N. Spergel and A. Vilenkin, Phys. Rev. D 34, 944 (1986).

[8] "Cosmic Strings and Other Topological Defects," A. Vilenkin and E.P.S. Shellard, C.U.P., 1994.

[9] A. Vilenkin and T. Vachaspati, Phys. Rev. Lett. 58, 1041 (1987).

[10] J. J. Blanco-Pillado and K. D. Olum, Nucl. Phys. B 599, 435 (2001)

[11] J. J. Blanco-Pillado, K. D. Olum and A. Vilenkin, Phys. Rev. D 63, 103513 (2001)

[12] C. J. A. Martins and E. P. S. Shellard, Phys. Rev. D 73, 043515 (2006)

[13] V. Vanchurin, K. D. Olum and A. Vilenkin, Phys. Rev. D 74, 063527 (2006)

[14] C. Ringeval, M. Sakellariadou and F. Bouchet, JCAP 0702, 023 (2007)

[15] The loop distribution is still under study e.g. [16]. In [17] the authors find that loops will only be of microscopic size, in which case radio emission is only possible from cusps on long strings. In [18], an important step is taken towards including radiation backreaction but the backreaction on the loop production function, which feeds into the loop distribution function, is ignored.

[16] F. Dubath, J. Polchinski and J. V. Rocha, arXiv:0711.0994 [astro-ph].

[17] G. Vincent, N. D. Antunes and M. Hindmarsh, Phys. Rev. Lett. 80, 2277 (1998)

[18] J. V. Rocha, Phys. Rev. Lett. 100, 071601 (2008)

[19] L. C. Lee and J. R. Jokipii, Ap. J. 206, 735 (1976).

[20] C. A. Katz, J. N. Hewitt, B. E. Corey and C. B. Moore, arXiv:astro-ph/0304260

[21] S. M. Barr and A. M. Matheson, Phys. Rev. D 36, 2905 (1987).

[22] S. M. Barr and A. M. Matheson, Phys. Lett. B 198, 146 (1987).

[23] F. Ferrer, H. Mathur, T. Vachaspati and G. D. Starkman, Phys. Rev. D 74, 025012 (2006)

[24] J. P. Ostriker, A. C. Thompson and E. Witten, Phys. Lett. B 180, 231 (1986).

[25] N. G. Sanchez and M. Signore, Phys. Lett. B 261, 21 (1991).

[26] T. Damour and A. Vilenkin, Phys. Rev. Lett. 85, 3761 (2000) X. Siemens, J. Creighton, I. Maor, S. Ray Majumder, K. Cannon and J. Read, Phys. Rev. D 73, 105001 (2006)

[27] F. Ferrer and T. Vachaspati, Phys. Rev. Lett. 95, 261302 (2005)

[28] D. Garfinkle and T. Vachaspati, Phys. Rev. D 36, 2229 (1987). 